Check for updates

Cite this: Chem. Sci., 2019, 10, 4905

๑ All publication charges for this article have been paid for by the Royal Society of Chemistry

\section{Bonding heterogeneity and lone pair induced anharmonicity resulted in ultralow thermal conductivity and promising thermoelectric properties in $\mathrm{n}$-type $\mathrm{AgPbBiSe}_{3} \dagger$}

\author{
Moinak Dutta, ${ }^{a}$ Koushik Pal, ${ }^{\mathrm{b}}$ Umesh V. Waghmare ${ }^{\mathrm{b}}$ and Kanishka Biswas (DD *a
}

Received 28th January 2019

Accepted 3rd April 2019

DOI: $10.1039 / \mathrm{c} 9 \mathrm{sc} 00485 \mathrm{~h}$

rsc.li/chemical-science

\begin{abstract}
Efficiency in generation and utilization of energy is highly dependent on materials that have the ability to amplify or hinder thermal conduction processes. A comprehensive understanding of the relationship between chemical bonding and structure impacting lattice waves (phonons) is essential to furnish compounds with ultralow lattice thermal conductivity $\left(\kappa_{\text {lat }}\right)$ for important applications such as thermoelectrics. Here, we demonstrate that the $n$-type rock-salt AgPbBiSe 3 exhibits an ultra-low $\kappa_{\text {lat }}$ of $0.5-0.4 \mathrm{~W} \mathrm{~m}^{-1} \mathrm{~K}^{-1}$ in the 290-820 $\mathrm{K}$ temperature range. We present detailed analysis to uncover the fundamental origin of such a low $\kappa_{\text {lat. }}$. First-principles calculations augmented with low temperature heat capacity measurements and the experimentally determined synchrotron X-ray pair distribution function (PDF) reveal bonding heterogeneity within the lattice and lone pair induced lattice anharmonicity. Both of these factors enhance the phonon-phonon scattering, and are thereby responsible for the suppressed $\kappa_{\text {lat. }}$. Further optimization of the thermoelectric properties was performed by aliovalent halide doping, and a thermoelectric figure of merit $(z T)$ of 0.8 at $814 \mathrm{~K}$ was achieved for $\mathrm{AgPbBiSe} \mathrm{P}_{2.97} \mathrm{I}_{0.03}$ which is remarkable among $n$-type Te free thermoelectrics.
\end{abstract}

\section{Introduction}

Almost all energy conversion processes liberate heat as their universal by-product with varying degrees. Minimizing this waste heat is thus of paramount importance, and a prime method for inhibiting such waste heat liberation is to have compounds that obstruct heat propagation. Hence compounds with innate low lattice thermal conductivity $\left(\kappa_{\text {lat }}\right)$ are an intriguing prospect in this regard and can be utilized in the field of thermoelectrics. ${ }^{1-3}$ Typically, in crystalline solids, phonons

${ }^{a}$ New Chemistry Unit, Jawaharlal Nehru Centre for Advanced Scientific Research (JNCASR), Jakkur P.O., Bangalore 560064, India. E-mail: kanishka@jncasr.ac.in ${ }^{b}$ Theoretical Sciences Unit, Jawaharlal Nehru Centre for Advanced Scientific Research (JNCASR), Jakkur P.O., Bangalore 560064, India

$\dagger$ Electronic supplementary information (ESI) available: Contains the method of refinement of PDF (Fig. S1), figures containing different unit cells (Fig. S2); phonon dispersion of different supercells (Fig. S3); low $T C_{\mathrm{p}}$ data (Fig. S4); $\kappa_{\text {diff }}$ values (Fig. S5); figures containing supercells, charge density and ELF of $\mathrm{AgPbBiSe}_{3}$ (Fig. S6); visualization of eigen vectors (Fig. S7); mode Gruneisen parameters (Fig. S8); phonon dispersion curves (Fig. S9); avoided acoustic-optical crossing (Fig. S10); temperature dependent $R_{\mathrm{w}}$ and lattice parameter, a (Fig. S11); local structure fit (Fig. S12), PXRD data (Fig. S13); band gaps (Fig. S14); atom projected electronic structure (Fig. S15); mobility vs. carrier plot (Fig. S16). Also contains tables of $C_{\mathrm{p}} / T$ vs. $T^{2}$ refined parameters (Table S1); LA and TA frequencies (Table S2); $U_{\text {iso }}$ values (Table S3); band-gap (Table S4); carrier conc. (n) vs. mobility $\left(\mu_{\mathrm{H}}\right)$ (Table S5). See DOI: $10.1039 / \mathrm{c} 9 \mathrm{sc} 00485 \mathrm{~h}$ and free charges are the primary carriers of heat. Since charge carriers are essential for efficient electrical conduction, inhibiting phonon propagation intrinsically is a primary method to reduce thermal conductivity, and is fostering significant attention towards developing materials with innately low $\kappa_{\text {lat }}{ }^{4}$

Investigations into the phonon-transport processes in solids with intrinsically low $\kappa_{\text {lat }}$ led to unearthing of various avenues to enhance the phonon scattering processes. Skutterudites ${ }^{5}$ and clathrates ${ }^{6}$ accommodate rattling guest atoms in hollow structural cages, thereby decreasing the $\kappa_{\text {lat }}$. Similarly, Zintl chalcogenides are known to contain weakly bonded rattling cations within a rigid covalent anionic sublattice..$^{7-9}$ Dynamic movement of cations in a crystalline matrix also causes lowering of $\kappa_{\text {lat }}$ via thermal damping effects. ${ }^{\mathbf{1 0 - 1 3}}$ Site disorder and stereochemically active $\mathrm{ns}^{2}$ lone electron pairs cause lattice anharmonicity, thus lowering the $\kappa_{\mathrm{lat} \cdot}{ }^{\mathbf{1 4 , 1 5}}$ Layered compounds are also known to have intrinsic ultralow $\kappa_{\text {lat }} \cdot{ }^{3,16}$ Several extrinsic approaches were also demonstrated to lower $\kappa_{\text {lat }}$ of compounds such as alloying, nano/meso structuring, etc., ${ }^{17-19}$ but they come with a caveat of having to compromise on the electrical mobility. Thus, to develop compounds with intrinsically low $\kappa_{\text {lat }}$, a thorough investigation and fundamental understanding of their chemistry including bonding, lattice dynamics and thermal transport mechanisms is essential.

Herein, we observe an ultralow $\kappa_{\text {lat }}$ of $c a .0 .5$ to $0.4 \mathrm{~W} \mathrm{~m}^{-1} \mathrm{~K}^{-1}$ for n-type $\mathrm{AgPbBiSe}$ in the temperature range of 290-823 K. 
$\mathrm{AgPbBiSe}_{3}$ adopts a rock-salt type structure where the $\mathrm{Ag} / \mathrm{Pb} / \mathrm{Bi}$ cations remain statistically disordered in the 4 a Wyckoff position while the $4 \mathrm{~b}$ Wyckoff site is occupied by the anion Se. ${ }^{20}$ The thermoelectric properties of a few compounds of $\mathrm{AgPbBiQ}_{3}(\mathrm{Q}=$ $\mathrm{S} / \mathrm{Se} / \mathrm{Te}$ ) were previously measured only at low temperatures (2$300 \mathrm{~K}$ ), but the $z T$ remains very low and the fundamental reasons behind the low $\kappa_{\text {lat }}$ were not explored in detail. ${ }^{20,21}$ To investigate the origin of the low $\kappa_{\text {lat }}$ of $\mathrm{AgPbBiSe}_{3}$, we have performed low temperature heat capacity measurements, which reveal Einstein modes and hence the presence of low lying optical phonons. Using first-principles (DFT) calculations, we show a remarkable crystallographic inhomogeneity associated with distinct bonding environments of the statistically disordered cations $(\mathrm{Ag} / \mathrm{Pb} / \mathrm{Bi})$ coordinated with anions $(\mathrm{Se})$. We also find high mode Gruneisen parameters $\left(r_{\mathrm{q} v} \sim 20\right)$, which are a measure of lattice anharmonicity, and trace their origin to the presence of $6 \mathrm{~s}^{2}$ lone pairs on $\mathrm{Pb}$ and $\mathrm{Bi}$. The pair distribution function (PDF) derived from the experimentally obtained synchrotron X-ray diffraction patterns enabled us to determine the bonding and lattice environment in terms of the nearest neighbour atoms in real space and obtain valuable microscopic insights into the mechanism behind the low $\kappa_{\text {lat }}$. Results of the PDF analysis corroborate our theoretical inferences of bonding heterogeneity and the presence of lone pairs, which are responsible for inhibition of the heat carrying acoustic phonons.

Demonstrating low thermal conductivity, which is a prerequisite to realization of high thermoelectric performance, we have optimised the thermoelectric figure of merit $(z T)$ of this ntype $\mathrm{AgPbBiSe}{ }_{3}$ via aliovalent halogen doping. We have observed a further reduction in the $\kappa_{\text {lat }}$ of $\mathrm{AgPbBiSe}_{3}$ to $0.23 \mathrm{~W} \mathrm{~m}^{-1} \mathrm{~K}^{-1}$ at $823 \mathrm{~K}$, which is attributed to point defect scattering. As a result, we report a two-fold increase in the $z T$ from 0.43 for pristine $\mathrm{AgPbBiSe}_{3}$ at $818 \mathrm{~K}$ to 0.8 for $\mathrm{AgPbBiSe}_{2.97} \mathrm{I}_{0.03}$ at $814 \mathrm{~K}$.

\section{Experimental section}

\section{Reagents}

Elemental silver (Sigma Aldrich, 99.99\%), lead (Alfa Aesar, 99.999\%), bismuth (Sigma Aldrich, 99.999\%), selenium (Alfa Aesar, 99.9999\%) and $\mathrm{BiX}_{3}(\mathrm{X}=\mathrm{Cl}$, Br, I; Sigma Aldrich, 99.99\%) were used as starting materials for the synthesis, without further purification.

\section{Synthesis}

A stoichiometric ratio of the reagents was used to prepare $\sim 10 \mathrm{~g}$ crystalline ingots of $\mathrm{AgPbBiSe}_{3}$ and $\mathrm{AgPbBiSe}_{3-y} \mathrm{X}_{y}(\mathrm{X}=\mathrm{Cl}, \mathrm{Br}, \mathrm{I}$ and $y=0.03$ for $\mathrm{Cl}$ and $\mathrm{I}, 0.02$ for $\mathrm{Br}$ ). The reagents were poured into $8 \mathrm{~mm}$ inner diameter quartz ampoules and subsequently flame-sealed under a high vacuum of $10^{-5}$ Torr. The vacuumsealed ampoules were then heated gradually to $723 \mathrm{~K}$ in $12 \mathrm{~h}$ to minimize Se evaporation, followed by heating up to $1223 \mathrm{~K}$ in $5 \mathrm{~h}$. They were then soaked at that temperature for $10 \mathrm{~h}$ with constant shaking to ensure homogeneity in the sample. The obtained melt compound was then cooled slowly to room temperature in $15 \mathrm{~h}$. Upon cooling the ingots obtained were cut into disk (having dimensions of $8 \mathrm{~mm}$ diameter and $2 \mathrm{~mm}$ thickness) and parallelepiped $\left(\sim 2 \times 3 \times 8 \mathrm{~mm}^{3}\right)$ shapes. The disk-shaped samples were used for thermal diffusivity measurements and the parallelepiped shaped samples were used for electrical transport measurements.

\section{Powder X-ray diffraction (PXRD)}

PXRD of the finely ground samples was performed using a Bruker D8 diffractometer with $\mathrm{Cu} \mathrm{K} \alpha(\lambda=1.5406 \AA)$ as the radiation source, for all the as-synthesized samples.

\section{Thermal conductivity}

Disk shaped samples were used for the measurement of thermal diffusivity $(D)$ via the laser flash technique using a Netzsch LFA 457 instrument under a $\mathrm{N}_{2}$ atmosphere. The obtained diffusivity was then used to measure the thermal conductivity of the samples using the formula $\kappa=D C_{\mathrm{p}} \rho$, where $\rho$ is the density of the measured samples and $C_{\mathrm{p}}$ is the heat capacity using the Dulong-Petit limit. All the measured samples have a density greater than $94 \%$. The error bars of the measured thermal conductivity data are within $10 \%$.

\section{Electrical properties}

Parallelepiped shaped samples were used for electrical transport measurements. All the samples were measured under a He atmosphere using an ULVAC-RIKO ZEM3 instrument. Both Seebeck coefficient and electrical conductivity were measured simultaneously within the temperature range $290-823 \mathrm{~K}$.

\section{Hall measurement}

Room temperature Hall measurement was performed using an in-house set up under a varying magnetic field of 0.0-0.57 T. The samples used were bar shaped and the current used was $75 \mathrm{~mA}$.

\section{Band gap measurement}

A finely ground sample was used to obtain the optical band gap of the synthesized samples. The optical band gap was estimated using a FT-IR Bruker IFS $66 \mathrm{~V} / \mathrm{S}$ spectrometer within the range of $6000-400 \mathrm{~cm}^{-1}$. Absorption $(\alpha / S)$ data were derived using the Kubelka-Munk equation, $\alpha / S=(1-R)^{2} /(2 R)$, where $R$ is the reflectance and $\alpha$ and $S$ correspond to absorption and scattering coefficient, respectively. The energy band gaps were deduced from the $\alpha / S$ vs. $E_{\mathrm{g}}(\mathrm{eV})$ plot.

\section{X-ray Pair Distribution Function (PDF)}

Finely ground $\mathrm{AgPbBiSe}{ }_{3}$ was used for the temperature dependent PDF. The experiment was performed at beamline P02.1, PETRA III, DESY, Hamburg. The data were obtained as a 2D .tiff image file which was then converted to a 1D .chi file using the software fit2D. ${ }^{22}$ The obtained .chi file where the intensities of the peaks were plotted against $Q$-space (in $\mathrm{nm}$ ) was converted into real space (as a .gr file) using the software PDFgetX2. ${ }^{23}$ The obtained .gr file was then refined using the software PDFgui. ${ }^{24}$ 


\section{Computational methods}

Our first-principles calculations are based on density functional theory (DFT) using the Vienna Ab initio Simulation Package (VASP). ${ }^{25,26}$ The potential energy of the interaction between the ionic core of an atom and valence electrons was treated within the projected augmented wave (PAW) ${ }^{27}$ method. The exchange and correlation energy of electrons was included within a generalized gradient approximated (GGA) ${ }^{28}$ functional parametrized by Perdew, Burke and Ernzerhof. ${ }^{29}$ Expansions of electronic wave functions on a plane wave basis were truncated with a cut-off energy of $520 \mathrm{eV}$. Brillouin Zone (BZ) integrations were sampled on a uniform mesh of $8 \times 8 \times 8 k$-points. The discontinuity in the occupation numbers of the electronic states near the gap was smeared with the Fermi-Dirac distribution functions with a broadening of $k_{\mathrm{B}} T=0.04 \mathrm{eV}$. To model $\mathrm{AgPbBiSe}_{3}$ (in which $\mathrm{Ag}, \mathrm{Pb}$ and $\mathrm{Bi}$ are statistically disordered at the cation sites) in our DFT calculations, we constructed an isotropic supercell containing 6 atoms ( 3 cations and 3 anions). This supercell basically consists of three primitive unit cells of a face centered cubic lattice. As there are three cations $\mathrm{Ag}, \mathrm{Pb}$, and $\mathrm{Bi}$ ) in the crystal structure, and three cation sites in our supercell, there would be a total of six possible configurations. After fully optimizing all the structures, we found them to be iso-energetic (the energy difference between any two structures is less than $10^{-5}$ eV f.u. ${ }^{-1}$ ). We used one of the structures to perform further calculations. We provide the lattice vectors, atomic positions and all six configurations of $\mathrm{AgPbBiSe}_{3}$ in Fig. S2 of the ESI. $\dagger$ As phonon frequencies obtained using this supercell revealed imaginary phonon modes at several points (including the zone center, i.e., $\Gamma$ ) in the Brillouin zone, we distorted the crystal structure by displacing atoms along the eigen vectors of the unstable phonon mode at $\Gamma$ and relaxed the atomic positions (the internal degrees of freedom of the supercell). We used this relaxed supercell (which stabilizes the $\Gamma$-point phonons) in further calculations to determine the electronic structure and lattice dynamic properties of $\mathrm{AgPbBiSe}_{3}$.

Phonon dispersion of $\mathrm{AgPbBiSe}{ }_{3}$ was determined using the finite-displacement method using Phonopy $^{30}$ and VASP calculations on a $(2 \times 2 \times 2)$ supercell containing 48 atoms. The Gruneisen parameter $(\gamma)$ measures the degree of lattice anharmonicity in a material. We estimated the mode Gruneisen parameter $\left(\gamma_{\mathrm{qv}}\right)$ of $\mathrm{AgPbBiSe} \mathrm{P}_{3}$ using a central difference formula and phonon frequencies calculated at volumes $\left(1.02 V_{0}\right.$ and $\left.0.98 V_{0}\right)$ around the equilibrium volume $\left(V_{0}\right)$ of the supercell. While we included the effect of spin-orbit coupling (SOC) in the calculations of the electronic structure, phonon frequencies were obtained without including the SOC in our calculations. To check the convergence of the phonon frequencies with respect to the supercell size, we used a $3 \times 3 \times 3$ supercell (with 162 atoms) and the same convergence criteria as above with a slightly reduced $k$-point grid $(2 \times 2 \times 2)$. The changes in the phonon frequencies are not very significant particularly for the acoustic phonon branches (Fig. S3, ESI $\dagger$ ) Hence, all further calculations were performed using the $(2 \times 2 \times 2)$ supercell.

\section{Results and discussion}

High quality pure crystalline ingots of $\mathrm{AgPbBiSe}_{3}$ were synthesized via a melting process under a high vacuum of $10^{-5}$ Torr. Fig. 1a shows Le Bail refinement of the powder-XRD data obtained using an in-house X-ray diffractometer $(\mathrm{Cu} \mathrm{K} \alpha ; \lambda=$ $1.5406 \AA$ A) with a cubic rock-salt model, which confirms that $\mathrm{AgPbBiSe}_{3}$ possesses a $\mathrm{NaCl}$ structure (space group, $\mathrm{Fm} \overline{3} \mathrm{~m}$ ). The anion $4 \mathrm{~b}$ Wyckoff site is occupied by the Se atoms whereas the cation $4 \mathrm{a}$ Wyckoff site is occupied by three cations in statistical disorder (i.e., Ag, Bi and $\mathrm{Pb}$ ) as shown in Fig. 1b. The presence of 3 cations at the $4 \mathrm{a}$ Wyckoff site results in a greater degree of cation disorder in the crystal system. The global structure is apparently similar to rich LAST $\left(\mathrm{AgPb}_{m} \mathrm{SbTe}_{2+m}\right.$ i.e. PbTe rich), ${ }^{31}$ where 3 cations $\mathrm{Ag} / \mathrm{Pb} / \mathrm{Sb}$ at the same site provided enhanced scattering of phonons. Here too in Te free $\mathrm{AgPbBiSe}_{3}$, the positionally disordered cations at the same position were expected to result in lowering of thermal conductivity to a certain extent via a site scattering mechanism. Fig. 2a depicts the temperature dependent total thermal conductivity $\left(\kappa_{\text {tot }}\right)$ and $\kappa_{\text {lat }}$ of $\mathrm{AgPbBiSe}_{3}$. The $\kappa_{\text {lat }}$ is found to be ultra-low and remains almost flat within the measured temperature range with values from $0.50 \mathrm{~W} \mathrm{~m}^{-1} \mathrm{~K}^{-1}$ at $296 \mathrm{~K}$ to $0.41 \mathrm{~W} \mathrm{~m}^{-1} \mathrm{~K}^{-1}$ at $818 \mathrm{~K}$. Although the value is ultra-low it is found to be higher than the minimum $\kappa_{\text {lat }}\left(0.206 \mathrm{~W} \mathrm{~m}^{-1} \mathrm{~K}^{-1}\right)$ derived using the diffusion model $^{32}$ as well as from Cahill's formulation $\left(0.327 \mathrm{~W} \mathrm{~m}^{-1}\right.$

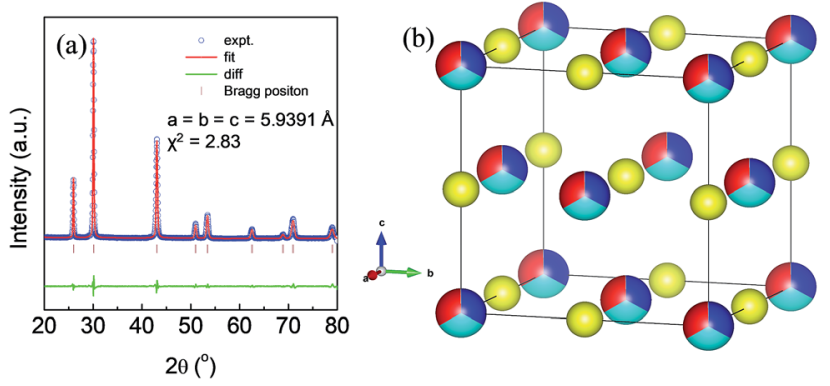

Fig. 1 (a) Le Bail refinement of the powder XRD pattern of $\mathrm{AgPbBiSe}_{3}$. (b) Rock-salt structure of the compound $\mathrm{AgPbBiSe}_{3}$. Red, sky blue, blue, and yellow atoms represent $\mathrm{Ag}, \mathrm{Pb}, \mathrm{Bi}$ and Se respectively.
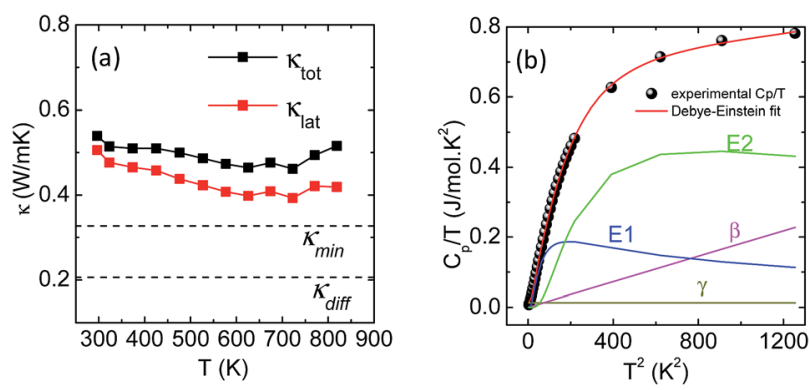

Fig. 2 (a) Total (black) and lattice (red) thermal conductivity of pristine $\mathrm{AgPbBiSe}_{3}$. (b) $C_{\mathrm{p}} / T$ vs. $T^{2}$ plot within the $2-30 \mathrm{~K}$ range. The red line is the fit produced using the combined Debye-Einstein model. The individual contributions from electronic $(\gamma)$, Debye $(\beta)$ and the two Einstein terms $\left(E_{1}\right.$ and $\left.E_{2}\right)$ are also plotted. 
$\left.\mathrm{K}^{-1}\right)^{33}$ which enhances the scope for further lowering of the $\kappa_{\text {lat }}$. The electronic contribution to the $\kappa_{\text {tot }}$ is found to be very low and is given in Fig. S4, ESI. $\dagger$ For a better comprehension of this underlying ultralow lattice conductivity in $\mathrm{AgPbBiSe}_{3}$, we have measured the low temperature heat capacity $C_{\mathrm{p}}(\sim 2 \mathrm{~K}$ to $\sim 200$ K; Fig. S5, ESI $\dagger$ ). Fig. $2 \mathrm{~b}$ shows the $C_{\mathrm{p}} / T$ vs. $T^{2}$ plot with temperatures ranging from $2 \mathrm{~K}$ to $30 \mathrm{~K}$. Modelling the plot using only Debye modes was insufficient; thus it was imperative to incorporate the Einstein oscillators for proper analysis of the data. The combined Debye-Einstein model ${ }^{\mathbf{8}, 10,34}$ (eqn (1)) is as follows.

$$
\frac{C_{\mathrm{p}}}{T}=r+\beta T^{2}+\sum_{n}\left(A_{n}\left(\theta_{E_{n}}\right)^{2}\left(T^{2}\right)^{-\frac{3}{2}} \frac{\mathrm{e}^{\frac{\theta_{E_{n}}}{T}}}{\left(\mathrm{e}^{\frac{\theta_{E_{n}}}{T}}-1\right)^{2}}\right)
$$

This combined Debye-Einstein model is composed of three terms. The temperature independent part of eqn (1), i.e., $r$, signifies the electronic contribution. The second term, $\beta$, corresponds to contributions arising from Debye modes where,

$$
\beta=B\left(12 \pi^{4} N_{\mathrm{A}} \kappa_{\mathrm{B}} / 5\right)\left(\theta_{\mathrm{D}}\right)^{-3}
$$

$N_{\mathrm{A}}, \kappa_{\mathrm{B}}$ and $\theta_{\mathrm{D}}$ represent the Avogadro number, Boltzmann constant and Debye temperature, respectively. The term $B$ in eqn (2) is given by $B=1-\sum_{n} A_{n} / 3 N R$, where $N$ and $R$ represent the number of atoms per formula unit and the universal gas constant (8.314 $\mathrm{J} \mathrm{mol}^{-1} \mathrm{~K}^{-1}$ ), respectively. The final term takes care of the Einstein oscillators, where $A_{n}$ is the Einstein prefactor to the $n^{\text {th }}$ Einstein mode $\left(\theta_{\mathrm{E} n}\right)$. A minimum of 2 Einstein modes along with the Debye modes were mandatory for a better fitting of the low temperature $C_{\mathrm{p}}$ data (Fig. 2b). The individual contributions of each term are also plotted in Fig. $2 \mathrm{~b}$. The fitting parameters that are obtained are provided in Table S1, ESI. $\dagger$ The Debye temperature $\left(\theta_{\mathrm{D}}\right)$ derived for $\mathrm{AgPbBiSe}{ }_{3}$ is found to be $172 \mathrm{~K}$. The characteristic temperature of the Einstein modes estimated from the fit are $\theta_{\mathrm{E} 1}=35.6017 \mathrm{~K}$ $\left(\sim 24 \mathrm{~cm}^{-1}\right)$ and $\theta_{\mathrm{E} 2}=74.47509 \mathrm{~K}\left(\sim 51 \mathrm{~cm}^{-1}\right)$ which indicate the presence of low-lying optical modes. These low lying optical modes are pivotal for low $\kappa_{\text {lat }}$ as they hinder the thermal flow via coupling with the heat-carrying acoustic phonons. We have determined the mean velocity of sound $\left(v_{\mathrm{s}}\right)$ using the formula $\kappa_{\mathrm{B}} \theta_{\mathrm{D}}=\hbar\left(6 \pi^{2} n\right)^{1 / 3} v_{\mathrm{s}},{ }^{32}$ where $n$ is the number density of atoms and $\hbar$ is the reduced Planck constant. The average or mean velocity of sound is derived to be $\sim 1710 \mathrm{~m} \mathrm{~s}^{-1}$.

To learn further about the bonding environment and atomic dynamics within the crystal structure and their role in suppressing the $\kappa_{\text {lat }}$ of $\mathrm{AgPbBiSe}{ }_{3}$, we now present the results of first-principles density functional theory (DFT) calculations using the Vienna Ab initio Simulation Package (VASP). ${ }^{25,26}$ We analyse the chemical bonding environment in $\mathrm{AgPbBiSe}_{3}$ with the real space charge density and electron localization function (ELF). Charge density analysis (Fig. S6b, ESI $\dagger$ ) reveals that electron clouds of more electropositive Bi atoms strongly overlap with Se, suggesting a strong covalent character of the bonding between them. On the other hand, the electron clouds around less electropositive $\mathrm{Ag}$ and $\mathrm{Pb}$ cations overlap relatively weakly with the anions, indicating weaker bonding. Coexistence of such strong and weak bonding and cationic disorder give rise to crystallographic heterogeneity, resulting in low lattice thermal conductivity in materials. ${ }^{7,8,16,35}$ To demonstrate the impact of bonding heterogeneity on the dynamics of the atoms, we calculated the potential energy (Fig. 3a) landscape of atomic displacements around their equilibrium positions in $x$ - and $z$-directions. A shallow potential well of displacements of $\mathrm{Ag}$ atoms (see Fig. 3a) signifies the fact that $\mathrm{Ag}$
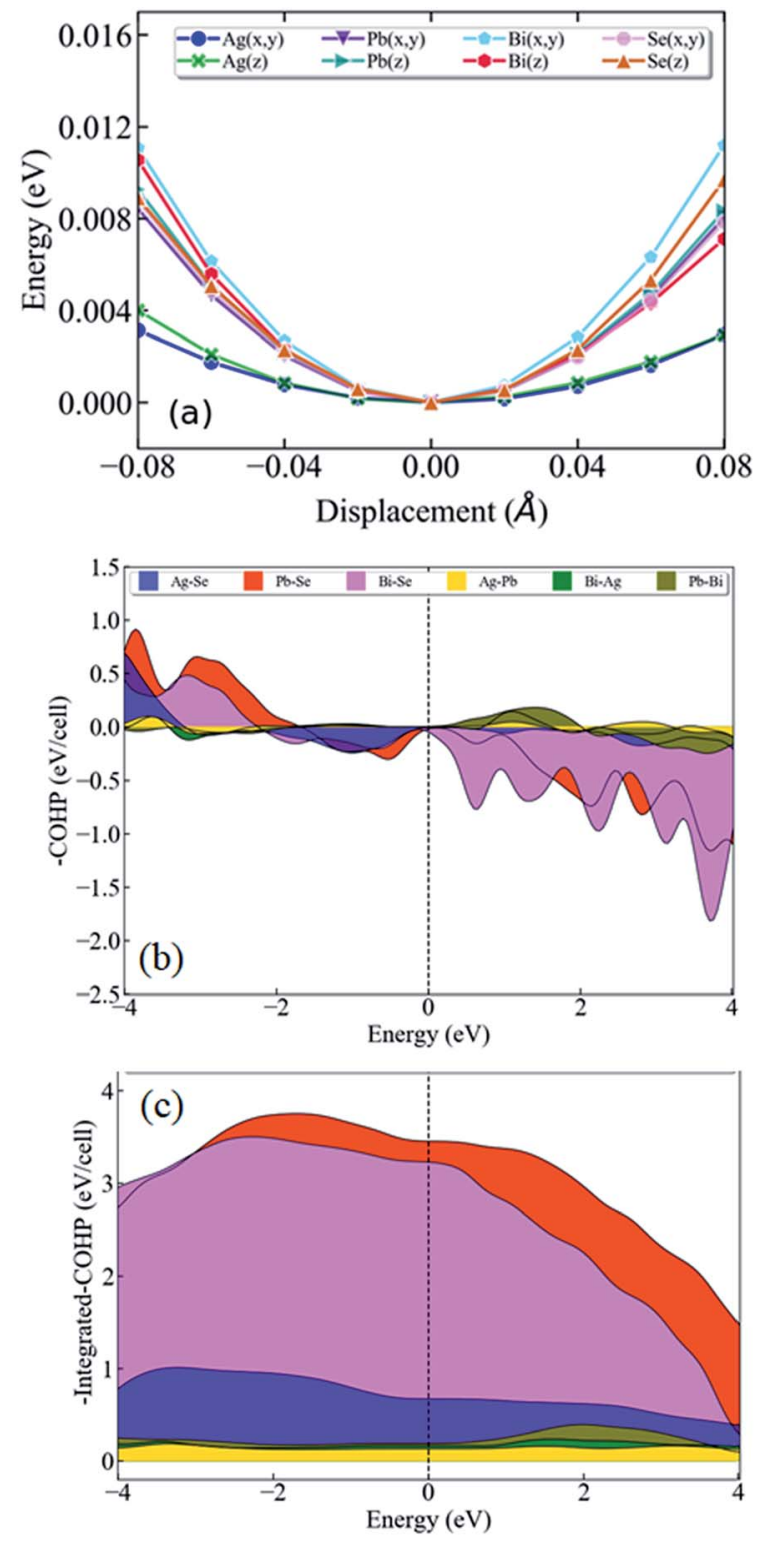

Fig. 3 (a) Potential energy curves of the constituent atoms in $\mathrm{AgPbBiSe}_{3}$ as a function of displacement around their equilibrium positions along the $x$ - and $z$-direction. (b) Crystal orbital Hamilton population (COHP) and (c) integrated $\mathrm{COHP}$ (iCOHP) for the nearest neighbor atomic pairs of $\mathrm{AgPbBiSe}_{3}$. The smaller peak in (b) below the Fermi level and smallest area under the curve in (c) of $\mathrm{Ag}$-Se interaction shows that the $\mathrm{Ag}$ atoms have the weakest bonding to the lattice. 
atoms are loosely bound to the lattice, and their vibrations result in greater dynamic structural disorder and entropy. Due to their relatively stronger bonding, $\mathrm{Bi}, \mathrm{Pb}$ and $\mathrm{Se}$ atoms have deeper potential energy wells than Ag (Fig. 3a). Larger amplitudes of atomic vibrations of $\mathrm{Ag}$ atoms than of $\mathrm{Pb}, \mathrm{Bi}$ and Se are also evident in the visualization of eigen vectors of the lowest energy optical phonons at the $\Gamma$ point (Fig. S7, ESI $\dagger$ ). The visualization of the eigen vectors at $30 \mathrm{~cm}^{-1}$ and $50 \mathrm{~cm}^{-1}$ (Fig. S7, ESI $\dagger$ ) shows a greater vibration of Ag, which indicates that $\mathrm{Ag}$ is loosely bound to the lattice as compared to other cations which leads to the origin of these low lying optical phonons. These low energy Ag vibrations can be compared to the low lying optical modes derived from low temperature heat capacity $\left(C_{\mathrm{p}}\right)$ data.

We now present the crystal orbital Hamilton population (COHP) analysis, in which the energy of electronic states of a crystal is partitioned into pairwise orbital interactions, enabling quantification of the role of atomic orbitals in chemical bonding. ${ }^{36,37}$ Moreover, the integrated COHP (iCOHP) gives the contribution of a chemical bond to the band energy, indicating its strength. ${ }^{38,39}$ Here, we have used the LOBSTER code ${ }^{36}$ COHP analysis of different nearest neighbor pairs of atoms of $\mathrm{AgPbBiSe}_{3}$. We find that the nearest neighbor Ag-Se interaction results in the weakest peak (Fig. 3b) in COHP curves below the Fermi level, showing weaker interaction of $\mathrm{Ag}$ than of the other cations. As expected, the integrated COHP for Ag-Se interaction is the smallest (see area under the curves in Fig. 3c) confirming the weakest binding of $\mathrm{Ag}$ atoms to the lattice of $\mathrm{AgPbBiSe}_{3}$.

Visualization of the electron localization function (Fig. S6c, ESI $\dagger$ ) shows the presence of lone pairs around $\mathrm{Bi}$ and $\mathrm{Pb}$ atoms. Lone pairs are known to play an important role in reducing the $\kappa_{\text {lat }}$ in many well-known thermoelectric materials. ${ }^{14,15}$ To quantify this effect of the lone pairs in $\mathrm{AgPbBiSe}_{3}$, we calculated the mode Gruneisen parameters $\left(r_{\mathrm{qv}}\right)$ which are a measure of the degree of anharmonicity in a lattice. They specifically give the third order interaction between phonons and acoustic waves (primary carriers of heat). Very high values of $r_{\mathrm{qv}}(\sim 20)$ of the lowest energy optical phonons and of phonons in the acoustic branches (Fig. S8, ESI $\dagger$ ) along several high symmetric directions (e.g., $\Gamma$ - $\mathrm{K} 1, \Gamma-\mathrm{K} 2$ and $\Gamma-\mathrm{K} 3)$ in the Brillouin zone indicate strong scattering of acoustic phonons by inhomogeneous strains associated with cationic disorder. Projected phonon density of states, DOS (Fig. 4a) reveals that low frequency optical modes
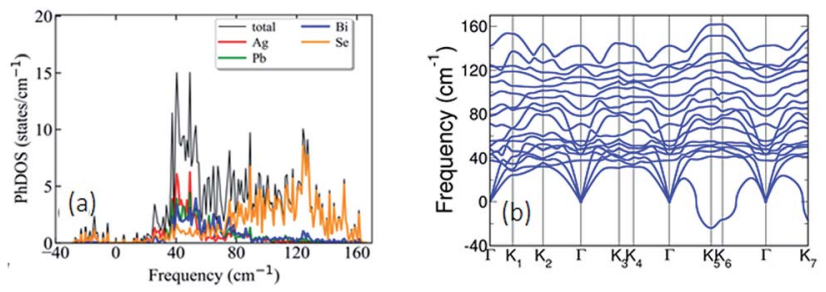

Fig. 4 (a) Atom projected phonon density of states reveals that low frequency optical modes $\left(<50 \mathrm{~cm}^{-1}\right)$ originate largely due to the vibrations of the $\mathrm{Ag}$ atoms followed by contributions from $\mathrm{Pb}$ and $\mathrm{Bi}$ atoms. (b) Phonon dispersion of $\mathrm{AgPbBiSe}_{3}$ showing that the acoustic branches have low cut-off frequencies $\left(<50 \mathrm{~cm}^{-1}\right)$. $\left(<50 \mathrm{~cm}^{-1}\right)$ are dominated by vibrations of $\mathrm{Ag}$ atoms followed by the contributions from $\mathrm{Pb}$ and $\mathrm{Bi}$ atoms. This is also evident in the atom projected phonon dispersion curves (Fig. S9, ESI $†$ ). The role of the cations in the optical-acoustic phonon scattering processes is evident from the avoided crossing between the branches of acoustic and low frequency optical modes in the phonon dispersion (Fig. S10, ESI†). Thus, lone pairs of Bi and $\mathrm{Pb}$ atoms cause strengthening of their bonds with Se by offcentering, and consequent weakening of $\mathrm{Ag}$-Se bonds. This results in a combination of chemical and structural heterogeneity at atomic scales, leading to suppression of relaxation times of acoustic phonon scattering and hence low $\kappa_{\text {lat }}$ of $\mathrm{AgPbBiSe}_{3}$.

Calculated phonon dispersion (Fig. $4 \mathrm{~b}$ ) shows that the cutoff frequencies of the acoustic modes are quite low $\left(<50 \mathrm{~cm}^{-1}\right)$, suggesting low sound velocity which further contributes to lowering the thermal conductivity in $\mathrm{AgPbBSe}_{3}$, through quadratic dependence (within the kinetic theory): $\kappa_{\mathrm{lat}}=$ $0.33 C_{\mathrm{v}} v_{\mathrm{s}}{ }^{2} \tau$. Here, $C_{\mathrm{v}}$ corresponds to the heat capacity at a constant volume, $v_{\mathrm{s}}$ is the average sound velocity and $\tau$ denotes the phonon relaxation time. In Table S2, ESI $\dagger$ the cutoff frequencies for the longitudinal (LA) and two transverse (TA1 and TA2) acoustic modes for the in-plane and out-of-plane directions in the Brillouin zone are listed. The low cut-off frequency points are due to the presence of heavy atoms and the bonding inhomogeneity amongst them. The average sound velocity $\left(v_{\mathrm{s}}\right)$ of $\mathrm{AgPbBiSe}_{3}$ is $1860 \mathrm{~m} \mathrm{~s}^{-1}$, calculated using $v_{\mathrm{s}}=\frac{1}{3}\left(\frac{1}{v_{1}}+\frac{2}{v_{\mathrm{t}}^{2}}\right)^{-\frac{1}{3}}$, which agrees well with the sound velocity $\left(1710 \mathrm{~m} \mathrm{~s}^{-1}\right)$ derived from the $C_{\mathrm{p}}$ measurements. We estimated the longitudinal $\left(v_{1}=3310 \mathrm{~m} \mathrm{~s}^{-1}\right)$ and transverse $\left(v_{\mathrm{t}}=\right.$ $1610 \mathrm{~m} \mathrm{~s}^{-1}$ ) sound velocities of this compound through the equations:

$$
v_{1}=\sqrt{\left(B+\frac{4}{3} G\right) / \rho}
$$

and

$$
v_{\mathrm{t}}=\sqrt{G / \rho},
$$

where $B, G, \rho$ denote the bulk modulus, shear modulus and density of the material, respectively.

In mixed-valence systems, phonon scattering is enhanced by the electron charge transfer which contributes to a further reduction of $\kappa_{\text {lat }}{ }^{20}$ To determine if there is any charge transfer occurring in $\mathrm{AgPbBiSe}_{3}$, we calculated the dynamical (born effective) charges $\left(Z^{*}\right)$, and found significant deviations from the nominal valence charges of each atom: $\left(\mathrm{Ag}: Z^{*} x x(y y)=2.3\right.$, $Z^{*}{ }_{z z}=2.1$; Pb: $Z^{*}{ }_{x x(y y)}=4.3, Z^{*}{ }_{z z}=5.1 ; \mathrm{Bi}: Z^{*}{ }_{x x(y y)}=6.4, Z_{z z}^{*}=$ 5.9; Se: $\left.Z_{x x(y y)}^{*}=-6, Z_{z z}^{*}=-1.6\right)$. Such anomalously large positive charges of the cations and negative charges of the anions indicate dynamic (hopping) transfer of electronic charge from cations to the anions during polar vibrations.

An in-depth experimental analysis of the structure for this ensuing low $\kappa_{\text {lat }}$ is complicated due to the presence of disordered cations which might induce lattice strain. This implies 
that the local bonding environment might be different from the average/global structure. Hence it is paramount to augment the traditional crystallographic measurements with local structure measuring techniques. The atomic pair distribution function (PDF) specializes in providing both diffuse scattering (which gives information regarding the structure at an atomic level) and Bragg scattering (which provides information regarding the global atomic structure), thus providing us microscopic insights into the role that the structure plays in reducing the thermal conductivity. Fig. 5a depicts the temperature dependent synchrotron X-ray PDF data from $100-400 \mathrm{~K}$ at an equal interval of $50 \mathrm{~K}$ as a function of $r$ (in $\AA$ ). A decrease in intensity and broadening of the peaks were observed, which is normally due to the enhanced thermal vibrations along high symmetry directions in the Brillouin zone with increasing temperature. The temperature dependent PDF data were fitted using the cubic model of $\mathrm{AgPbBiSe}_{3}$ and the goodness of fit $\left(R_{\mathrm{w}}\right)$ is shown in Fig. S11a, ESI. $\dagger$

The temperature dependent lattice parameter, $a$ is given in Fig. S11b, ESI $\dagger$ and is shown to linearly increase with temperature. The thermal parameter $\left(U_{\text {iso }}\right)$ obtained from the refinement (please see the ESI $\dagger$ under the section "methods" for additional details regarding the refinement) clearly shows higher ADP values for $\mathrm{Ag}$ than for $\mathrm{Se}$, with $\mathrm{Bi}$ and $\mathrm{Pb}$ having the lowest ADP values (Fig. 5b). ADPs of $\mathrm{Pb}$ are actually almost same as those of $\mathrm{Bi}$ and as a result ADPs of Bi overlap with those of $\mathrm{Pb}$. The similar ADP values of both $\mathrm{Pb}$ and $\mathrm{Bi}$ are due to their similar size and the values are presented in Table S3, ESI. $\dagger$ The high $\mathrm{ADP}$ values of $\mathrm{Ag}$ compared to those of other atoms corroborate the theoretical prediction that the $\mathrm{Ag}$ atoms are loosely bonded in the crystal lattice and contribute to the anharmonicity, thereby suppressing $\kappa_{\text {lat }}$. Parts a and b of Fig. 6 represent the first peak (which provides information of the nearest atomatom correlations) and total peak fitting using the cubic model, respectively. The blue circles correspond to the experimental data at a temperature of $100 \mathrm{~K}$ while the red line represents the fitted data. The difference between the fitted and experimental data is shown in green. It can be clearly seen that although the total average structure gives a good fit to the cubic model, the first peak which corresponds to the nearest anion to cation distance (i.e., at a distance of $a / 2$ ) could not be properly fitted using the cubic model. To understand the local dynamics of the
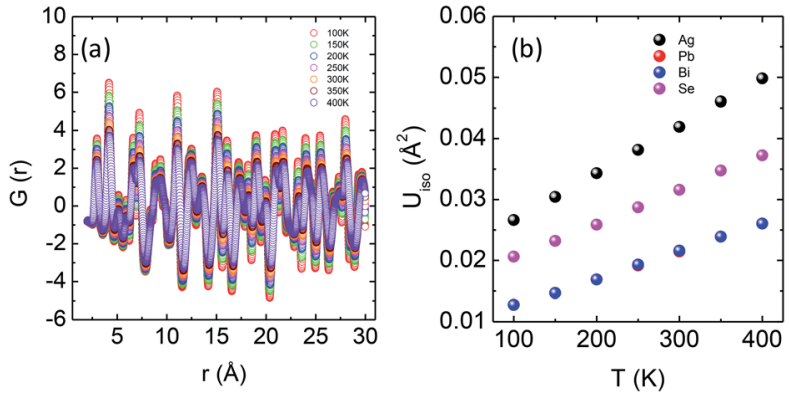

Fig. 5 (a) Temperature dependent atomic PDF $G(r)$ plots of $\mathrm{AgPbBiSe}_{3}$ plotted against real space. (b) Thermal ADPs of individual Ag, Pb, Bi and Se atoms plotted against temperature.
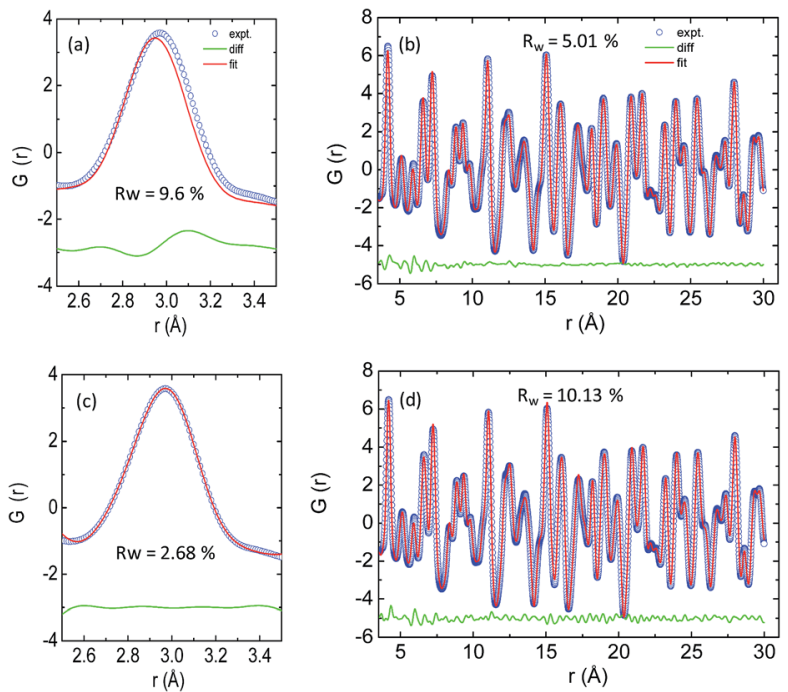

Fig. 6 X-ray PDF data: (a) first peak and (b) total peak fitting using the cubic model. (c) First peak and (d) total peak fitting using the locally distorted model. The experimental data used at a temperature of $100 \mathrm{~K}$.

structure and subsequently their role in the low $\kappa_{\text {lat }}$ of the compound, we tried to refine the first peak as it tells us about any local distortion in the system. We have observed that the best fit is possible when the cations are displaced from their original place in the $\langle 111\rangle$ direction (see the ESI $\dagger$ for details).

Although the first peak gave a very good account of the local distortion, the overall fitting was worse compared to the cubic model as evident from the corresponding $R_{\mathrm{w}}$ values. Parts $\mathrm{c}$ and d of Fig. 6 show the fitting using the locally distorted cubic structure for the first atom-atom correlation and the total fit, respectively. It has been observed that by distorting $\mathrm{Pb}$ and $\mathrm{Bi}$ to a higher degree compared to Ag, the nature of the first peak fits better. Fig. S12, ESI $\dagger$ shows the first peak fitting at $400 \mathrm{~K}$ which can be compared with the first peak fitting at $100 \mathrm{~K}$ (Fig. 6c). The fitting of the first peak at these two temperatures $(100 \mathrm{~K}$ and 400 $\mathrm{K})$ was possible by off-centering $\mathrm{Pb}$ and Bi by $0.047 \AA$ at $100 \mathrm{~K}$ and by $0.21 \AA$ at $400 \mathrm{~K}$. Ag gets off-centered to a lesser degree from $0.018 \AA$ to $0.06 \AA$ at $100 \mathrm{~K}$ and $400 \mathrm{~K}$, respectively. The higher magnitude of off-centering in $\mathrm{Pb}$ and $\mathrm{Bi}$ might be due to the presence of $6 \mathrm{~s}^{2}$ lone pair electrons, and is similar to that of $\mathrm{PbTe}$ and $\mathrm{PbS}$, where it was argued that the stereochemical activity of $6 \mathrm{~s}^{2}$ lone pairs of $\mathrm{Pb}^{2+}$ contributed to the off-centering of $\mathrm{Pb}$ and subsequent suppression of the $\kappa_{\mathrm{lat}}{ }^{40}$ Thus, lone pairs of $\mathrm{Bi}^{3+}$ and $\mathrm{Pb}^{2+}$ can be presumed to play a prominent role in suppressing $\kappa_{\text {lat }}$ via inducing strong lattice anharmonicity.

Hence, it can be argued that the low $\kappa_{\text {lat }}$ in $\mathrm{AgPbBiSe}_{3}$ is a result of bonding heterogeneity whereby $\mathrm{Ag}$, being loosely bonded, is effective in scattering heat carrying phonons as well as lone pairs of electrons on $\mathrm{Pb}$ and $\mathrm{Bi}$ which induce anharmonicity in the lattice.

Since materials with low $\kappa_{\text {lat }}$ are useful in waste heat management, we have further improved the thermoelectric figure of merit in this n-type $\mathrm{AgPbBiSe}_{3}$ via aliovalent halogen doping. The thermoelectric figure of merit $(z T)$ of thermoelectric materials is determined using the formula 


$$
z T=\frac{\sigma S^{2}}{\kappa} T
$$

where, $\sigma$ and $S$ represent the electrical conductivity and Seebeck coefficient, respectively. $\kappa$ is the thermal conductivity which constitutes electronic $\left(\kappa_{\mathrm{el}}\right)$ and lattice $\left(\kappa_{\text {lat }}\right)$ contributions, i.e., $\kappa$ $=\kappa_{\mathrm{el}}+\kappa_{\text {lat }}$. PXRD patterns of all the halide doped compounds can be indexed according to the cubic rock-salt structure (Fig. S13, ESI $\dagger$ ) and shows no presence of second phase peaks within the detection limit.

Band gap measurements reveal that n-type $\mathrm{AgPbBiSe}{ }_{3}$ has a band gap $\left(E_{\mathrm{g}}\right)$ of $0.46 \mathrm{eV}$ which is consistent with the earlier reported $E_{\mathrm{g}}$ of $0.48 \mathrm{eV}^{20}$ Halide doping in $\mathrm{AgPbBiSe}_{3}$ does not show any significant change in band gap as shown in Fig. S14, ESI $\dagger$ and the band gap values are given in Table S4, ESI. $\dagger$ The electronic structure (Fig. 7a) of $\mathrm{AgPbBiSe}_{3}$ determined from DFT calculations including spin-orbit coupling reveals that it possesses a small $E_{\mathrm{g}}$ of $0.14 \mathrm{eV}$. Theoretical calculations are known to underestimate band gaps of compounds and thus shows a lower value compared to the experimentally observed band gap. The projected electronic structure (Fig. S15, ESI $\dagger$ ) and density of states (Fig. 7b) show that while valence bands just below the Fermi level are primarily constituted of orbital contributions of the Se atoms, the conduction bands above the Fermi level have contributions from all the atoms.

Electrical transport measurement has been performed for all the samples within the temperature range of 290-823 K. Electrical conductivity $(\sigma)$, as expected, increases with halogen doping as it imparts an extra electron and can be understood via simple valence counting. For pristine $\mathrm{AgPbBiSe}{ }_{3}$, the electrical conductivity at room temperature is $63 \mathrm{~S} \mathrm{~cm}^{-1}$ and rises slightly to $72 \mathrm{~S} \mathrm{~cm}^{-1}$ at $818 \mathrm{~K}$ (Fig. 8a). Cl doped $\mathrm{AgPbBiSe}{ }_{2.97} \mathrm{Cl}_{0.03}$ contributes the most to enhancing the electrical properties. Doping with $\mathrm{Cl}$ increases the electrical conductivity to $263 \mathrm{~S} \mathrm{~cm}^{-1}$ at $297 \mathrm{~K}$ and $240 \mathrm{~S} \mathrm{~cm}^{-1}$ at $813 \mathrm{~K}$ compared to the pristine $\mathrm{AgPbBiSe}$, which has a considerably lower $\sigma$ (Fig. 8a). Doping with $\mathrm{Br}$ and I increases the electrical conductivity too but the increase is considerably less compared to that with $\mathrm{Cl}$ (Fig. 8a). The difference in electrical conductivity with various

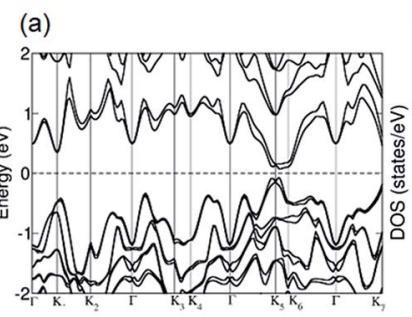

(b)

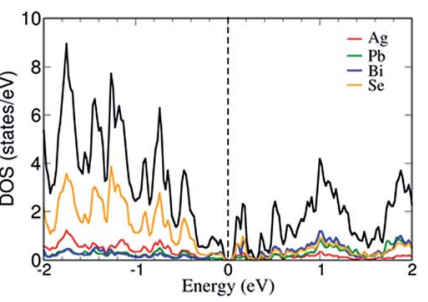

Fig. 7 (a) Electronic structure of $\mathrm{AgPbBiSe}_{3}$ calculated with spin-orbit coupling (SOC) shows that it is a small band gap semiconductor with multiple bands near the Fermi level. (b) Projected density of states reveals that the valence and conduction bands around the Fermi level mainly constitute bonding and anti-bonding states arising from the hybridization of atomic orbitals between $\mathrm{Bi}$ and $\mathrm{Se}$ atoms. While $\mathrm{Ag}$ atoms have the weakest bonding in the lattice, the bonding between $\mathrm{Pb}$ and $\mathrm{Se}$ atoms are also relatively weak.
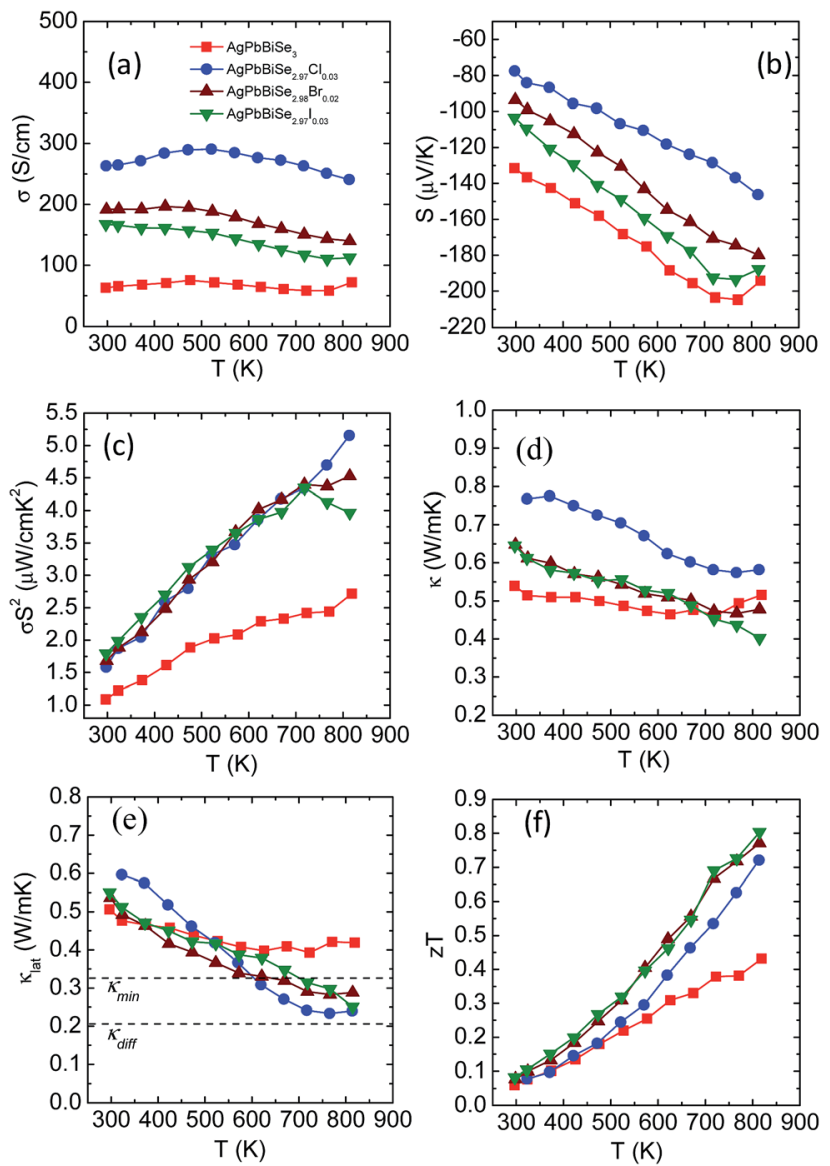

Fig. 8 Temperature dependent (a) electrical conductivity, (b) Seebeck co-efficient, (c) power factor, (d) thermal conductivity, (e) lattice thermal conductivity and ( $f$ ) thermoelectric figure of merit $(z T)$ for pristine and halogen doped $\mathrm{AgPbBiSe}_{3}$.

doping is due to the respective size of the halogens. $\mathrm{Cl}$, for which the size is close to that of Se as compared to $\mathrm{Br}$ and $\mathrm{I}$, moves easily into the Se lattice and imparts the extra electron with better efficiency than $\mathrm{Br}$ or I. The carrier concentration and mobility of the doped $\mathrm{AgPbBiSe}{ }_{3}$ are presented in Table S5, ESI $\dagger$ and resemble the electrical conductivity trend. The carrier concentration of pristine $\mathrm{AgPbBiSe}_{3}$ at room temperature is 1.44 $\times 10^{18} \mathrm{~cm}^{-3}$, which increases to $1.34 \times 10^{19} \mathrm{~cm}^{-3}$ for AgPbBiSe ${ }_{2.97} \mathrm{Cl}_{0.03}$ (Table S5, ESI $\dagger$ ). Similarly, the Seebeck coefficient $(S)$ value measured for pristine $\mathrm{AgPbBiSe}_{3}$ is $-131 \mu \mathrm{V} \mathrm{K}^{-1}$ at $296 \mathrm{~K}$ which increases to $-204 \mu \mathrm{V} \mathrm{K}^{-1}$ at $770 \mathrm{~K}$ (Fig. 8b). The negative sign in the Seebeck coefficient value corresponds to $n$ type conduction which is also corroborated from the Hall measurement data. The $S$ value decreases with the doping concentration which is in line with the electrical conductivity and carrier concentration $(n)$ trend. The electrical conductivity and Seebeck co-efficient result in a power factor of 1.09 $\mu \mathrm{W} \mathrm{cm}{ }^{-1} \mathrm{~K}^{-2}$ at $296 \mathrm{~K}$ which increases to $2.71 \mu \mathrm{W} \mathrm{cm}{ }^{-1} \mathrm{~K}^{-2}$ at $818 \mathrm{~K}$ for pristine $\mathrm{AgPbBiSe}_{3}$ (Fig. 8c). The power factor of the doped compounds is understandably higher compared to that of the pristine sample with a maximum power factor of $c a .5 .15$ $\mu \mathrm{W} \mathrm{cm}^{-1} \mathrm{~K}^{-2}$ at $813 \mathrm{~K}$ for the $\mathrm{Cl}$ doped $\mathrm{AgPbBiSe}{ }_{2.97} \mathrm{Cl}_{0.03}$ (Fig. 8c). 
Fig. 8d and e depict the total thermal conductivity and lattice thermal conductivity of the doped compounds. It has been observed that the lattice thermal conductivity of the halogen doped $\mathrm{AgPbBiSe}_{3}$ decreases from $0.6 \mathrm{~W} \mathrm{~m}^{-1} \mathrm{~K}^{-1}$ at $290 \mathrm{~K}$ to around $0.23 \mathrm{~W} \mathrm{~m}^{-1} \mathrm{~K}^{-1}$ at $823 \mathrm{~K}$. The decrease in the lattice thermal conductivity compared to that of the pristine counterpart is due to point defects arising from halogen doping which scatter the high frequency phonons. The carrier $(n) v s$. mobility $\left(\mu_{\mathrm{H}}\right)$ plot (Fig. S16, ESI $\dagger$ ) shows that the mobility decreases with the increase in carrier concentration. We noticed that $\mu_{\mathrm{H}} \sim n^{-1 /}$ ${ }^{3}$ dependency provides a correct description of their relationship, which indicates that the acoustic phonon scattering due to charge carriers is the major scattering process in these halogen doped samples. ${ }^{\mathbf{4 1}}$

The thermoelectric figure of merit $(z T)$ which takes into account the power factor and the thermal conductivity is found to be 0.43 for pristine $\mathrm{AgPbBiSe}_{3}$ at $818 \mathrm{~K}$ and it increases almost two-fold to 0.8 at $814 \mathrm{~K}$ in $\mathrm{AgPbBiSe}_{2.97} \mathrm{I}_{0.03}$, which is considerably high in n-type Te free compounds (Fig. 8f). In comparison, n-type $\mathrm{AgBiSe}_{2}$ has a peak $z T$ of $\sim 0.9$ at $810 \mathrm{~K}$ (ref. $42)$ and n-type $(\mathrm{GeSe})_{0.50}\left(\mathrm{AgBiSe}_{2}\right)_{0.50}$ exhibits a $z T$ of 0.45 at 677 K. ${ }^{43}$ n-type BiSe achieved a $z T$ of 0.8 at $425 \mathrm{~K}$ via Sb substitution. ${ }^{16}$ Polycrystalline $\mathrm{n}$-type PbSe on the other hand achieved a peak $z T$ of $\sim 1.8$ at $723 \mathrm{~K}$ for $\mathrm{PbSe}_{0.998} \mathrm{Br}_{0.002}-2 \% \mathrm{Cu}_{2} \mathrm{Se}^{44}$ Although PbSe based n-type thermoelectrics provide a much better $z T$, it is worth noting that it took almost a decade to achieve these types of $z T$ values in PbSe. Our compound $\mathrm{AgPbBiSe}_{3}$ on the other hand is in its nascent stage and with further optimization of its electronic properties, a higher $z T$ can also be achieved for $\mathrm{AgPbBiSe}_{3}$.

\section{Conclusions}

$\mathrm{AgPbBiSe}_{3}$ exhibits ultralow lattice thermal conductivity in the temperature range $290-823 \mathrm{~K}$. Low temperature heat capacity experiments proved the presence of low lying optical phonon modes which are pivotal in scattering the heat-carrying acoustic phonons. Theoretical probing into the phonon mechanism using first principles DFT calculations revealed a high degree of anharmonicity arising from the chemical disorder of cations. The compound possesses bonding inhomogeneity wherein the $\mathrm{Ag}$ atoms are weakly bonded as compared to $\mathrm{Pb}$ and $\mathrm{Bi}$ in the lattice. Electron localization function analysis reveals the presence of $6 \mathrm{~s}^{2}$ lone pairs in $\mathrm{Pb}$ and $\mathrm{Bi}$ which induce strong variation in the chemical bonding of cations, and hence the lattice anharmonicity. Thus, the synergistic presence of bonding heterogeneity and lattice anharmonicity arising from $6 \mathrm{~s}^{2}$ lone pairs of $\mathrm{Bi}$ and $\mathrm{Pb}$ fosters low lattice thermal conductivity of $0.5 \mathrm{~W} \mathrm{~m}^{-1} \mathrm{~K}^{-1}$ at $296 \mathrm{~K}$ which further decreases to $c a .0 .4 \mathrm{~W} \mathrm{~m}^{-1}$ $\mathrm{K}^{-1}$ at $818 \mathrm{~K}$. Further reduction of this low thermal conductivity and enhancement in the power factor in the n-type $\mathrm{AgPbBiSe}{ }_{3}$ compound have been performed by halogen substitution, and we achieved a high $z T$ of 0.8 at $814 \mathrm{~K}$ in $\mathrm{AgPbBiSe}_{2.97} \mathrm{I}_{0.03}$. We hope that this fundamental understanding of the lattice thermal conductivity and its interplay with the structural aspects, chemical bonding and lattice dynamics will guide the designing and furnishing of new materials having low intrinsic thermal conductivity.

\section{Conflicts of interest}

The authors declare no competing financial interests.

\section{Acknowledgements}

The work is supported by the Sheikh Saqr Laboratory and by the SERB (EMR/2016/651). M. D. acknowledges the University Grants Commission (UGC) for the research fellowship. UVW acknowledges support from a J C Bose National Fellowship of the Department of Science and Technology, Govt. of India and Sheikh Saqr Fellowship. Synchrotron X-ray measurements for this research were carried out at the light source PETRAIII at the DESY, a member of the Helmholtz Association (HGF). We would like to thank Dr J. C. Bednarcik for assistance at beamline P02.1, Petra III. Financial support from the Department of Science and Technology (Government of India) provided within the framework of the India@DESY collaboration is gratefully acknowledged.

\section{References}

1 G. Tan, L.-D. Zhao and M. G. Kanatzidis, Chem. Rev., 2016, 116, 12123-12149.

2 L.-D. Zhao, V. P. Dravid and M. G. Kanatzidis, Energy Environ. Sci., 2014, 7, 251-268.

3 C. Chang, M. Wu, D. He, Y. Pei, C.-F. Wu, X. Wu, H. Yu, F. Zhu, K. Wang, Y. Chen, L. Huang, J.-F. Li, J. He and L.-D. Zhao, Science, 2018, 360, 778.

4 M. K. Jana and K. Biswas, ACS Energy Lett., 2018, 3, 13151324.

5 X. Shi, J. Yang, J. R. Salvador, M. Chi, J. Y. Cho, H. Wang, S. Bai, J. Yang, W. Zhang and L. Chen, J. Am. Chem. Soc., 2011, 133, 7837-7846.

6 T. Takabatake, K. Suekuni, T. Nakayama and E. Kaneshita, Rev. Mod. Phys., 2014, 86, 669-716.

7 M. K. Jana, K. Pal, U. V. Waghmare and K. Biswas, Angew. Chem., Int. Ed., 2016, 55, 7792-7796.

8 M. K. Jana, K. Pal, A. Warankar, P. Mandal, U. V. Waghmare and K. Biswas, J. Am. Chem. Soc., 2017, 139, 4350-4353.

9 S. Mukhopadhyay, D. S. Parker, B. C. Sales, A. A. Puretzky, M. A. McGuire and L. Lindsay, Science, 2018, 360, 1455.

10 H. Liu, X. Shi, F. Xu, L. Zhang, W. Zhang, L. Chen, Q. Li, C. Uher, T. Day and G. J. Snyder, Nat. Mater., 2012, 11, 422. 11 S. Roychowdhury, M. K. Jana, J. Pan, S. N. Guin, D. Sanyal, U. V. Waghmare and K. Biswas, Angew. Chem., Int. Ed., 2018, 57, 4043-4047.

12 S. N. Guin, J. Pan, A. Bhowmik, D. Sanyal, U. V. Waghmare and K. Biswas, J. Am. Chem. Soc., 2014, 136, 12712-12720.

13 B. Li, H. Wang, Y. Kawakita, Q. Zhang, M. Feygenson, H. L. Yu, D. Wu, K. Ohara, T. Kikuchi, K. Shibata, T. Yamada, X. K. Ning, Y. Chen, J. Q. He, D. Vaknin, R. Q. Wu, K. Nakajima and M. G. Kanatzidis, Nat. Mater., 2018, 17, 226-230. 
14 S. N. Guin, A. Chatterjee, D. S. Negi, R. Datta and K. Biswas, Energy Environ. Sci., 2013, 6, 2603-2608.

15 D. T. Morelli, V. Jovovic and J. P. Heremans, Phys. Rev. Lett., 2008, 101, 035901.

16 M. Samanta, K. Pal, P. Pal, U. V. Waghmare and K. Biswas, J. Am. Chem. Soc., 2018, 140, 5866-5872.

17 K. Biswas, J. He, I. D. Blum, C.-I. Wu, T. P. Hogan, D. N. Seidman, V. P. Dravid and M. G. Kanatzidis, Nature, 2012, 489, 414.

18 B. Poudel, Q. Hao, Y. Ma, Y. Lan, A. Minnich, B. Yu, X. Yan, D. Wang, A. Muto, D. Vashaee, X. Chen, J. Liu, M. S. Dresselhaus, G. Chen and Z. Ren, Science, 2008, 320, 634.

19 M. Samanta and K. Biswas, J. Am. Chem. Soc., 2017, 139, 9382-9391.

20 S. Sportouch, M. Basteat, P. Brazis, J. Ireland, C. R. Kannewurf, C. Uher and M. G. Kanatzidis, Mater. Res. Soc. Symp. Proc., 1999, 545, 123-130.

21 D. P. Spitzer, J. Phys. Chem. Solids, 1970, 31, 19-40.

22 A. P. Hammersley, S. O. Svensson, M. Hanfland, A. N. Fitch and D. Hausermann, High Pressure Res., 1996, 14, 235-248.

23 X. Qiu, J. W. Thompson and S. J. L. Billinge, J. Appl. Crystallogr., 2004, 37, 678.

24 C. L. Farrow, P. Juhas, J. W. Liu, D. Bryndin, E. S. Božin, J. Bloch, T. Proffen and S. J. L. Billinge, J. Phys.: Condens. Matter, 2007, 19, 335219.

25 G. Kresse and J. Hafner, Phys. Rev. B: Condens. Matter Mater. Phys., 1993, 48, 13115-13118.

26 G. Kresse and J. Furthmüller, Comput. Mater. Sci., 1996, 6, 15-50.

27 P. E. Blöchl, Phys. Rev. B: Condens. Matter Mater. Phys., 1994, 50, 17953-17979.

28 X. Hua, X. Chen and W. A. Goddard, Phys. Rev. B: Condens. Matter Mater. Phys., 1997, 55, 16103-16109.

29 J. P. Perdew, K. Burke and M. Ernzerhof, Phys. Rev. Lett., 1996, 77, 3865-3868.
30 A. Togo, F. Oba and I. Tanaka, Phys. Rev. B: Condens. Matter Mater. Phys., 2008, 78, 134106.

31 K. F. Hsu, S. Loo, F. Guo, W. Chen, J. S. Dyck, C. Uher, T. Hogan, E. K. Polychroniadis and M. G. Kanatzidis, Science, 2004, 303, 818.

32 M. T. Agne, R. Hanus and G. J. Snyder, Energy Environ. Sci., 2018, 11, 609-616.

33 D. G. Cahill, S. K. Watson and R. O. Pohl, Phys. Rev. B: Condens. Matter Mater. Phys., 1992, 46, 6131-6140.

34 H. Liu, J. Yang, X. Shi, S. A. Danilkin, D. Yu, C. Wang, W. Zhang and L. Chen, Journal of Materiomics, 2016, 2, 187-195.

35 K. Pal, J. He and C. Wolverton, Chem. Mater., 2018, 30, 77607768.

36 R. Dronskowski and P. E. Bloechl, J. Phys. Chem., 1993, 97, 8617-8624.

37 V. L. Deringer, A. L. Tchougréeff and R. Dronskowski, J. Phys. Chem. A, 2011, 115, 5461-5466.

38 S. Maintz, V. L. Deringer, A. L. Tchougréeff and R. Dronskowski, J. Comput. Chem., 2013, 34, 2557-2567.

39 A. Lücke, U. Gerstmann, T. D. Kühne and W. G. Schmidt, J. Comput. Chem., 2017, 38, 2276-2282.

40 E. S. Božin, C. D. Malliakas, P. Souvatzis, T. Proffen, N. A. Spaldin, M. G. Kanatzidis and S. J. L. Billinge, Science, 2010, 330, 1660.

41 A. F. May, J.-P. Fleurial and G. J. Snyder, Phys. Rev. B: Condens. Matter Mater. Phys., 2008, 78, 125205.

42 S. N. Guin, V. Srihari and K. Biswas, J. Mater. Chem. A, 2015, 3, 648-655.

43 S. Roychowdhury, T. Ghosh, R. Arora, U. V. Waghmare and K. Biswas, Angew. Chem., Int. Ed., 2018, 57, 15167-15171.

44 C. Zhou, Y. Yu, Y. K. Lee, O. Cojocaru-Mirédin, B. Yoo, S.-P. Cho, J. Im, M. Wuttig, T. Hyeon and I. Chung, J. Am. Chem. Soc., 2018, 140, 15535-15545. 